\title{
Thermal effect on unsteady flow of a dusty visco-elastic fluid between two parallel plates under different pressure gradients
}

\author{
K.R. Madhura ${ }^{1}$ and G. Kalpana ${ }^{2}$ \\ ${ }^{1}$ Department of Mathematics, East West Institute of Technology, Bangalore 560091, Karnataka, India \\ ${ }^{2}$ Department of Mathematics, Sri Krishna Institute of Technology, Bangalore 560090, Karnataka, India \\ Corresponding authors E-mail: madhurakrmadhu@gmail.com, g.kalpana006@gmail.com
}

\begin{abstract}
This article describes the effect of thermal diffusion on a flow of dusty viscous incompressible fluid which is electrically conducting and the flow is between two vertically heated, porous, parallel plates with heat source or sink. Variable separable and Laplace transform techniques are used to attain the solution of the mathematical equations. The effect of the temperature is examined for different values of Prandtl number $\left(P_{r}\right)$. The consequences of the concentration are observed for different values of thermal diffusion parameter $\left(T_{d}\right)$. The velocity profile for the flow of fluid and the dust particles are discussed for different values of $T_{d}$ and chemical reaction parameter $\left(c_{r}\right)$. In addition to that, the solutions are exhibited graphically.
\end{abstract}

Keywords: Chemical reaction, concentration, dusty fluid flow, exponential and linear pressure gradient, temperature, thermal diffusion, velocity profile.

\section{Introduction}

The short-lived caloric theory of heat is the origin of most heat transfer terminology used today. The study of thermal diffusion has a broad application area ranging from biological systems to common household appliances, residential and commercial buildings, industrial processes, electronic devices and food processing. The driving force for heat transfer is the temperature difference. Temperature is a measure of "heat concentration" and thus a high temperature region as one that has a high heat concentration. Therefore, heat transferred from the more concentrated regions to the less concentrated ones.

Chemical reaction can be codified as either heterogeneous or homogeneous processes. The study of thermal diffusion and chemical reaction has vast applications in many areas like sustain plasma confinement for controlled thermo nuclear fusion, liquid metal cooling of nuclear reactions and electromagnetic casting of metals. Paul. L. Chamber and Jonathan D. Young [1] have investigated a certain special class of homogeneous volume reactions in flow systems. P.G. Saffman [2] has studied the stability of laminar flow of a dusty gas. The laminar flow of a dusty gas between two rotating cylinders have studied by D.H. Michael and P.W. Norey [3]. A. Rapus and C.P. Perdikis [4] have got the solution for oscillatory flow through a porous medium in the presence of free convective flow.

D. Kumar and R.K. Srivastava [6] have worked on the effects of chemical reaction on MHD flow of dusty visco-elastic (Walter's liquid model-B) liquid with heat source/sink. I.U. Mbeledogu and A. Ogulu [7] have examined heat and mass transfer of an unsteady MHD natural convection flow of a rotating fluid past a vertical porous plate in the presence of radiactive heat transfer. P.M. Patil and P.S. Kulkarni [8] have found the effects of chemical reaction on free convective flow of a polar fluid through a porous medium in the presence of internal heat generation. Thermal diffusion and thermo effect on combined heat and mass transfer of a steady MHD convective and slip flow due to a rotating disk with viscous dissipation and ohmic heating have inspected by E. Osalusi, J. Side and R. Harris [9]. Ahmed A. Afify [10] has discussed MHD free convective heat and mass transfer over a stretching surface considering suction or injection under the effects of thermal-diffusion and diffusion thermo. 
O.A. Beg, A.Y. Bakier and V.R. Prasad [11] have explained numerical study of free convection magneto hydrodynamic heat and mass transfer from a stretching surface to a saturated porous medium with soret and dufour effects. K.R. Madhura, B.J. Gireesha and C.S. Bagewadi [12] have studied the flow of an unsteady dusty fluid through porous media in a channel of triangular cross-section. M.M. Nandeppanavar, M. Subhas Abel and JagadishTawade [13] have considered the heat transfer to visco elastic fluid (of Walter's liquid B) with combined effects of non-uniform source/sink, work done by elastic deformation. Heat transfer characteristics are examined for different kinds of boundary heating, namely prescribed surface temperature and prescribed wall heat flux.

Om Prakash, Devendra Kumar and Y.K. Dwivedi [14] have obtained the effects of thermal diffusion and chemical reaction on MHD flow of dusty visco-elastic (Walter's liquid model-B) fluid. R. Sharma, R. Bhargava and P. Bhargava [15] have scrutinized a numerical solution of steady MHD convection heat and mass transfer on a semi infinite vertical porous moving plate using element free Galerkin method. B.J. Gireesha, K.R. Madhura and C.S. Bagewadi [16] have crammed the flow of an unsteady dusty fluid through porous media between horizontal plate and a long wavy wall.

\section{Equations of motion}

Consider an unsteady visco-elastic incompressible fluid which is electrically conducting. The flow is between two vertically heated parallel plates with heat source or sink. The dust particles are uniformly distributed in a porous medium. The governing equations are given by

$\frac{\partial u}{\partial t}=-\frac{1}{\rho} \frac{\partial P}{\partial x}+g \beta\left(T-T_{0}\right)+g \beta\left(C-C_{0}\right)+\gamma\left(1-\lambda \frac{\partial}{\partial t}\right) \frac{\partial^{2} u}{\partial y^{2}}+\frac{k N}{\rho}(v-u)-\frac{\gamma}{k} u$,

$\frac{\partial v}{\partial t}=\frac{k}{m}(u-v)$

$\frac{\partial T}{\partial t}=\frac{K_{T}}{\rho C_{p}} \frac{\partial^{2} T}{\partial y^{2}}-\frac{Q_{1}}{\rho C_{p}}\left(T-T_{0}\right)$

$\frac{\partial C}{\partial t}=D \frac{\partial^{2} C}{\partial y^{2}}-\alpha\left(C-C_{0}\right)+D_{T} \frac{\partial^{2} T}{\partial y^{2}}$

The initial and boundary conditions are given by

i. when $t=0, u=0=v, T=T_{0}$ for $y \in(-h, h)$

ii. when $t>0, u=0=v, T=T_{0}+\left(T_{w}-T_{0}\right)\left(1-e^{-a t}\right)$ and $C=C_{0}+\left(C_{w}-C_{0}\right)\left(1-e^{-a t}\right)$ for $y=-h$ and $y=h$,

where

$u$ - Velocity of the fluid phase,

$t$ - Time,

$\rho$-Density of the fluid,

$P$ - Pressure of the fluid,

$x$-Co-ordinate axis in the direction of the flow,

$g$ - Acceleration due to gravity,

$\beta$-Volumetric thermal expansion coefficient,

$T$ - Temperature of the fluid,

$T_{0}$ - Initial temperature,

$C$-Concentration of the fluid,

$C_{0}-$ Initial uniform concentration at $T_{0}$, 
$\gamma$ - Kinematic viscosity,

$\lambda$ - Mean free path of the diffusing particles,

$y$-Co-ordinate axis as in normal to the plate,

$k$-Dimensionless chemical reaction parameter,

$N$ - Number density of the dust particles,

$v$-Velocity of the dust phase,

$m$ - Mass of the dust particles,

$K_{T}$ - Thermal conductivity coefficient of the fluid,

$C_{p}-$ Specific heat at constant pressure,

$Q_{1}$ - Volumetric rate of heat generation or absorption,

$D$ - Mass diffusivity (Mass diffusion rate),

$\alpha-$ Chemical reaction parameter,

$D_{T}$ - Coefficient of thermal diffusion.

\section{Formulation and solution of the problem}

Consider an unsteady laminar flow of an incompressible viscous fluid with uniform distribution of dust particles between two infinite moving plates separated by a distance ' $2 h$ ' in the absence of body force. The flow is due to the influence of time dependent pressure gradient and motion of plates. Both the fluid and the dust particle clouds are supposed to be static at the beginning. The dust particles are assumed to be spherical in shape and uniform in size. The number density of the dust particles is taken as a constant throughout the flow. Originally, when $t \leq 0$ the temperature and concentration of the dusty fluid is $T_{0}$ and $C_{0}$ respectively. When $t>0$, the temperature and concentration elevated to $T_{w}$ and $C_{w}$ respectively. Under these assumptions the flow will be a parallel flow in which the streamlines are along the $\mathrm{x}$-axis as shown below:

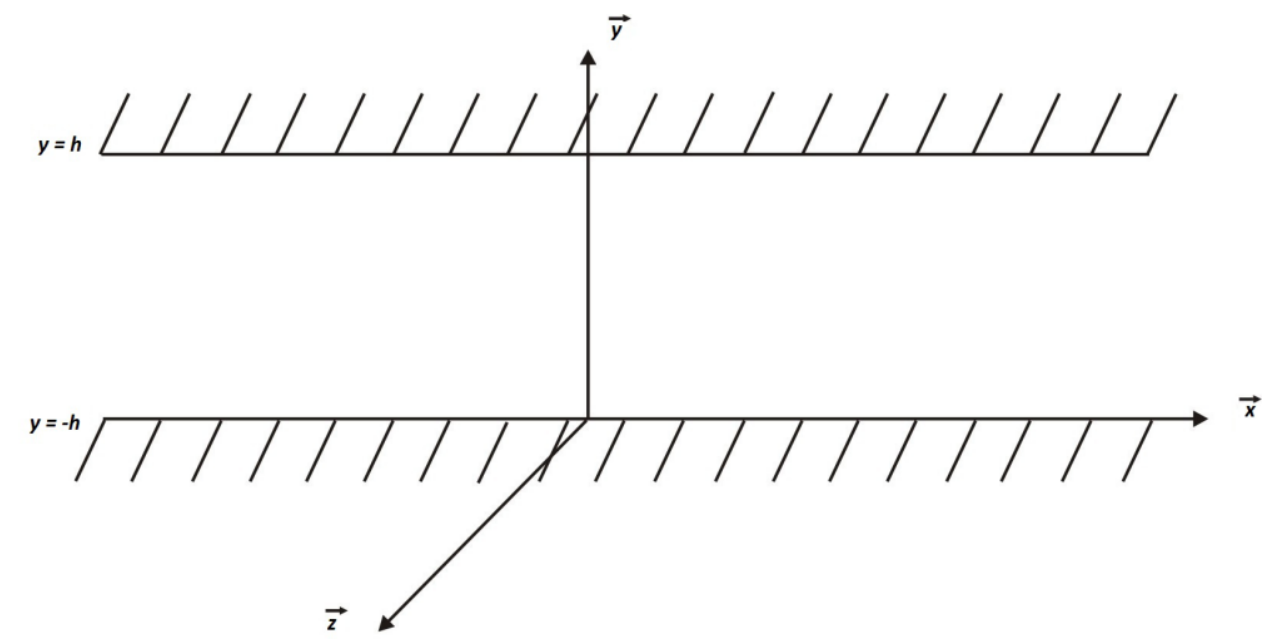

Fig. 1: Geometry of the flow

For the above prescribed flow, the velocities of fluid and the dust are of the form

$$
\vec{u}=u \vec{s}, \quad \vec{v}=v \vec{s} .
$$

The problem can be non-dimensionalised by substituting the following non-dimensional quantities 


$$
\begin{array}{ll}
P^{*}=\frac{P}{\gamma \rho}, & y^{*}=\frac{y}{h}, \quad x^{*}=\frac{x}{h}, \quad u^{*}=\frac{u}{h}, \quad v^{*}=\frac{v}{h}, \quad t^{*}=\frac{\gamma t}{h^{2}}, \\
a^{*}=\frac{a h^{2}}{\gamma}, & T^{*}=\frac{T-T_{0}}{T_{w}-T_{0}} \quad \text { and } \quad C^{*}=\frac{C-C_{0}}{C_{w}-C_{0}} .
\end{array}
$$

The equations (2.1), (2.2), (2.3) and (2.4) are reduced to

$\frac{\partial u}{\partial t}=-\frac{\partial P}{\partial x}+G_{r} T+G_{m} C+\left(1-V_{e} \frac{\partial}{\partial t}\right) \frac{\partial^{2} u}{\partial y^{2}}+\frac{l}{\tau}(v-u)-\frac{u}{M}$,

$\frac{\partial v}{\partial t}=\frac{1}{\tau}(u-v)$

$\frac{\partial^{2} T}{\partial y^{2}}-P_{r} \frac{\partial T}{\partial t}-H_{s} T=0$

$\frac{\partial^{2} C}{\partial y^{2}}-S_{c} \frac{\partial C}{\partial t}-c_{r} S_{c} C+T_{d} \frac{\partial^{2} T}{\partial y^{2}}=0$

The initial and boundary conditions reduced to

i. when $t=0, u=0=v, T=0$ for $y \epsilon(-1,1)$

ii. when $t>0, u=0=v, T=\left(1-e^{-a t}\right)$ and $C=\left(1-e^{-a t}\right)$ for $y=-1$ and $y=1$

where

Grashof number: $G_{r}=\frac{g \beta h\left(T_{w}-T_{0}\right)}{\gamma}$,

Modified grashof number: $G_{m}=\frac{g \beta h\left(C_{w}-C_{0}\right)}{\gamma}$,

Visco-elastic parameter: $V_{e}=\frac{\gamma \lambda}{h^{2}}$,

Mass concentration of dust particle: $l=\frac{m N}{\rho}$,

Relaxation time parameter: $\tau=\frac{m \gamma}{k h^{2}}$,

Prandtl number: $P_{r}=\frac{\mu c_{p}}{K_{T}}$,

Heat source or sink parameter: $H_{S}=\frac{Q_{1} h^{2}}{K_{T}}$,

Schmidt number: $S_{c}=\frac{\gamma}{D}$,

Dimensionless chemical reaction parameter: $c_{r}=\frac{\alpha h^{2}}{\gamma}$,

Thermal diffusion parameter: $T_{d}=\frac{D_{T}}{D}\left(\frac{T_{w}-T_{0}}{C_{w}-C_{0}}\right)$,

Viscosity of the fluid: $\mu=\gamma \rho$ and

Chemical reaction parameter: $M=\frac{K}{h^{2}}$. 
On solving the equation (3.3) using the variable separable method, the temperature is attained as

$T=\left(1-e^{-a t}\right) \frac{\cosh \left(m_{1} y\right)}{\cosh \left(m_{1}\right)}$

where $m_{1}=\sqrt{c_{1} P_{r} H_{s}}, c_{1}>0$ is the variable separable constant. Substituting the equation (3.5) in (3.4), the equation reduced to

$$
\frac{\partial^{2} C}{\partial y^{2}}-S_{c} \frac{\partial C}{\partial t}-c_{r} S_{c} C=-m_{1}{ }^{2} T_{d}\left(1-e^{-a t}\right) \frac{\cosh \left(m_{1} y\right)}{\cosh \left(m_{1}\right)}
$$

The solution of the above equation is as follows:

$$
C=\left(1-e^{-a t}\right) \frac{\cosh \left(m_{2} y\right)}{\cosh \left(m_{2}\right)}+\left(\mathrm{R}_{1}+\mathrm{R}_{2} \mathrm{e}^{-\mathrm{at}}\right)\left(\frac{\cosh \left(m_{2} y\right)}{\cosh \left(m_{2}\right)}-\frac{\cosh \left(m_{1} y\right)}{\cosh \left(m_{1}\right)}\right)
$$

where $m_{2}$ is the variable separable constant. On substituting the equation (3.5) and (3.6) in equation (3.1) which gives,

$$
\begin{aligned}
\frac{\partial u}{\partial t}= & -\frac{\partial P}{\partial x}+G_{r}\left(1-\mathrm{e}^{-\mathrm{at}}\right) \frac{\cosh \left(m_{1} y\right)}{\cosh \left(m_{1}\right)}+G_{m}\left(1-\mathrm{e}^{-\mathrm{at}}\right) \frac{\cosh \left(m_{2} y\right)}{\cosh \left(m_{2}\right)}+G_{m}\left(\mathrm{R}_{1}+\mathrm{R}_{2} \mathrm{e}^{-\mathrm{at}}\right) \\
& \times\left(\frac{\cosh \left(m_{2} y\right)}{\cosh \left(m_{2}\right)}-\frac{\cosh \left(m_{1} y\right)}{\cosh \left(m_{1}\right)}\right)+\left(1-V_{e} \frac{\partial}{\partial t}\right) \frac{\partial^{2} u}{\partial y^{2}}+\frac{l}{\tau}(v-u)-\frac{u}{M}
\end{aligned}
$$

Let $P_{0}(t)$ be the time dependent pressure gradient to be impressed on the system for $\mathrm{t}>0$. So we can write

$$
-\frac{\partial P}{\partial x}=P_{0}(t)
$$

Laplace transformation of $u$ and $v$ is given by

$L[u(t)]=\int_{0}^{\infty} e^{-s t} u(t) d t \quad$ and $\quad L[v(t)]=\int_{0}^{\infty} e^{-s t} v(t) d t$.

Applying the Laplace transformation to the equation (3.7) and (3.2) and to the boundary conditions, one can get

$$
\begin{aligned}
s \bar{u}= & P(s)+G_{r}\left(\frac{1}{s}-\frac{1}{s+a}\right) \frac{\cosh \left(m_{1} y\right)}{\cosh \left(m_{1}\right)}+G_{m}\left(\frac{1}{s}-\frac{1}{s+a}\right) \frac{\cosh \left(m_{2} y\right)}{\cosh \left(m_{2}\right)}+G_{m}\left(\frac{\mathrm{R}_{1}}{s}+\frac{\mathrm{R}_{2}}{s+a}\right) \\
& \times\left(\frac{\cosh \left(m_{2} y\right)}{\cosh \left(m_{2}\right)}-\frac{\cosh \left(m_{1} y\right)}{\cosh \left(m_{1}\right)}\right)+\left(1-V_{e} s\right) \frac{d^{2} \bar{u}}{d y^{2}}+\frac{l}{\tau}(\bar{v}-\bar{u})-\frac{\bar{u}}{M}, \\
s \bar{v}= & \frac{1}{\tau}(\bar{u}-\bar{v}), \\
\bar{u}=0 & =\bar{v} \text { for } y \epsilon(-1,1),
\end{aligned}
$$

where $P(s)$ is the Laplace transform of $P_{0}(t)$. 
$\bar{v}=\frac{\bar{u}}{1+s \tau}$

Using the equation (3.12), $\bar{v}$ is eliminated from the equation (3.9) and the differential equation is of the form

$$
\begin{aligned}
\frac{d^{2} \bar{u}}{d y^{2}}-Q^{2} \bar{u}= & -\frac{1}{1-V_{e} s}\left[P(s)+\left(\frac{1}{s}-\frac{1}{s+a}\right)\left(\frac{G_{r} \cosh \left(m_{1} y\right)}{\cosh \left(m_{1}\right)}+\frac{G_{m} \cosh \left(m_{2} y\right)}{\cosh \left(m_{2}\right)}\right)\right. \\
& \left.+G_{m}\left(\frac{\mathrm{R}_{1}}{s}+\frac{\mathrm{R}_{2}}{s+a}\right)\left(\frac{\cosh \left(m_{2} y\right)}{\cosh \left(m_{2}\right)}-\frac{\cosh \left(m_{1} y\right)}{\cosh \left(m_{1}\right)}\right)\right],
\end{aligned}
$$

where $Q^{2}=\frac{1}{1-V_{e} s}\left(s+\frac{s l}{1+s \tau}+\frac{1}{M}\right)$.

\section{Case - 1:}

Let $P_{0}(t)=a_{0} e^{-a_{1} t}$, where $a_{0}$ and $a_{1}$ are constants. The velocities of fluid and dust particles are obtained by solving the equation (3.13) and (3.12) subjected to the boundary conditions (3.11) as follows

$$
\begin{aligned}
\bar{u}= & \frac{a_{0}}{\left(s+a_{1}\right)\left(1-V_{e} s\right) Q^{2}}\left(1-\frac{\cosh (Q y)}{\cosh (Q)}\right)+\frac{1}{\left(1-V_{e} s\right)\left(m_{1}^{2}-Q^{2}\right)}\left(\frac{\cosh (Q y)}{\cosh (Q)}-\frac{\cosh \left(m_{1} y\right)}{\cosh \left(m_{1}\right)}\right) \\
& \times\left(\frac{G_{r}-\mathrm{R}_{1} G_{m}}{\mathrm{~s}}-\frac{G_{r}+\mathrm{R}_{2} G_{m}}{\mathrm{~s}+\mathrm{a}}\right)+\frac{G_{m}}{\left(1-V_{e} s\right)\left(m_{2}{ }^{2}-Q^{2}\right)}\left(\frac{\cosh (Q y)}{\cosh (Q)}-\frac{\cosh \left(m_{2} y\right)}{\cosh \left(m_{2}\right)}\right) \\
& \times\left(\frac{1+\mathrm{R}_{1}}{\mathrm{~s}}-\frac{1-\mathrm{R}_{2}}{\mathrm{~s}+\mathrm{a}}\right)
\end{aligned}
$$

and

$$
\begin{aligned}
\bar{v}= & \frac{a_{0}}{(1+s \tau)\left(s+a_{1}\right)\left(1-V_{e} s\right) Q^{2}}\left(1-\frac{\cosh (Q y)}{\cosh (Q)}\right)+\frac{1}{(1+s \tau)\left(1-V_{e} s\right)\left(m_{1}{ }^{2}-Q^{2}\right)} \\
& \times\left(\frac{\cosh (Q y)}{\cosh (Q)}-\frac{\cosh \left(m_{1} y\right)}{\cosh \left(m_{1}\right)}\right)\left(\frac{G_{r}-\mathrm{R}_{1} G_{m}}{\mathrm{~s}}-\frac{G_{r}+\mathrm{R}_{2} G_{m}}{\mathrm{~s}+\mathrm{a}}\right)+\frac{G_{m}}{(1+s \tau)\left(1-V_{e} s\right)\left(m_{2}{ }^{2}-Q^{2}\right)} \\
& \times\left(\frac{\cosh (Q y)}{\cosh (Q)}-\frac{\cosh \left(m_{2} y\right)}{\cosh \left(m_{2}\right)}\right)\left(\frac{1+\mathrm{R}_{1}}{\mathrm{~s}}-\frac{1-\mathrm{R}_{2}}{\mathrm{~s}+\mathrm{a}}\right)
\end{aligned}
$$

Applying the inverse Laplace transform to the equations (3.14) and (3.15), one can obtain $u$ and $v$ as

$$
\begin{aligned}
u= & \frac{a_{0} \mathrm{e}^{-\mathrm{a}_{1} \mathrm{t}}}{\alpha_{1}^{2}\left(1+\mathrm{a}_{1} V_{e}\right)}\left(1-\frac{\cosh \left(\alpha_{1} y\right)}{\cosh \left(\alpha_{1}\right)}\right)+\frac{4 M a_{0}}{\pi} \sum_{n=0}^{\infty} \frac{\cos \left(\frac{2 n+1}{2} \pi y\right)}{(-1)^{n}(2 n+1)}\left\{\frac{1}{\left(y_{1}+a_{1}\right)}\right. \\
& \left.\times \frac{e^{y_{1} t}\left(1+y_{1} \tau\right)^{2}\left(1-V_{e} y_{1}\right)}{\left[\left(1+y_{1} \tau\right)^{2}\left(M+V_{e}\right)+l M\left(1+\tau V_{e} y_{1}{ }^{2}\right)\right]}+\frac{e^{y_{2} t}\left(1+y_{2} \tau\right)^{2}\left(1-V_{e} y_{2}\right)}{\left(y_{2}+a_{1}\right)\left[\left(1+y_{2} \tau\right)^{2}\left(M+V_{e}\right)+l M\left(1+\tau V_{e} y_{2}{ }^{2}\right)\right]}\right\} \\
& +\pi M\left(G_{r}-\mathrm{R}_{1} G_{m}\right) \sum_{n=0}^{\infty} \frac{(2 n+1) \cos \left(\frac{2 n+1}{2} \pi y\right)}{(-1)^{n}\left[m_{1}{ }^{2}+\left(\frac{2 n+1}{2} \pi\right)^{2}\right]}\left\{\frac{e^{y_{1} t}\left(1+y_{1} \tau\right)^{2}\left(1-V_{e} y_{1}\right)}{y_{1}\left[\left(1+y_{1} \tau\right)^{2}\left(M+V_{e}\right)+l M\left(1+\tau V_{e} y_{1}{ }^{2}\right)\right]}\right. \\
& \left.+\frac{e^{y_{2} t}\left(1+y_{2} \tau\right)^{2}\left(1-V_{e} y_{2}\right)}{y_{2}\left[\left(1+y_{2} \tau\right)^{2}\left(M+V_{e}\right)+l M\left(1+\tau V_{e} y_{2}{ }^{2}\right)\right]}\right\}+\frac{\left(G_{r}-\mathrm{R}_{1} G_{m}\right)}{\left(m_{1}{ }^{2}-\alpha_{2}{ }^{2}\right)}\left(\frac{\cosh \left(\alpha_{2} y\right)}{\cosh \left(\alpha_{2}\right)}-\frac{\cosh \left(m_{1} y\right)}{\cosh \left(m_{1}\right)}\right) \\
& -\pi M\left(G_{r}+\mathrm{R}_{2} G_{m}\right) \sum_{n=0}^{\infty} \frac{(2 n+1) \cos \left(\frac{2 n+1}{2} \pi y\right)}{(-1)^{n}\left[m_{1}{ }^{2}+\left(\frac{2 n+1}{2} \pi\right)^{2}\right]}\left\{\frac{e^{y_{1} t}\left(1+y_{1} \tau\right)^{2}\left(1-V_{e} y_{1}\right)}{\left(y_{1}+a\right)\left[\left(1+y_{1} \tau\right)^{2}\left(M+V_{e}\right)+l M\left(1+\tau V_{e} y_{1}{ }^{2}\right)\right]}\right.
\end{aligned}
$$




$$
\begin{aligned}
& \left.+\frac{e^{y_{2} t}\left(1+y_{2} \tau\right)^{2}\left(1-V_{e} y_{2}\right)}{\left(y_{2}+a\right)\left[\left(1+y_{2} \tau\right)^{2}\left(M+V_{e}\right)+l M\left(1+\tau V_{e} y_{2}^{2}\right)\right]}\right\}-\frac{\left(G_{r}+\mathrm{R}_{2} G_{m}\right) e^{-a t}}{\left(1+a V_{e}\right)\left(m_{1}^{2}-\alpha_{3}^{2}\right)}\left(\frac{\cosh \left(\alpha_{3} y\right)}{\cosh \left(\alpha_{3}\right)}-\frac{\cosh \left(m_{1} y\right)}{\cosh \left(m_{1}\right)}\right) \\
& +\frac{G_{m}\left(1+\mathrm{R}_{1}\right)}{\left(m_{2}{ }^{2}-\alpha_{2}{ }^{2}\right)}\left(\frac{\cosh \left(\alpha_{2} y\right)}{\cosh \left(\alpha_{2}\right)}-\frac{\cosh \left(m_{2} y\right)}{\cosh \left(m_{2}\right)}\right)+\pi M G_{m}\left(1+\mathrm{R}_{1}\right) \sum_{n=0}^{\infty} \frac{(2 n+1) \cos \left(\frac{2 n+1}{2} \pi y\right)}{(-1)^{n}\left[m_{2}^{2}+\left(\frac{2 n+1}{2} \pi\right)^{2}\right]} \\
& \times\left\{\frac{e^{y_{1} t}\left(1+y_{1} \tau\right)^{2}\left(1-V_{e} y_{1}\right)}{y_{1}\left[\left(1+y_{1} \tau\right)^{2}\left(M+V_{e}\right)+l M\left(1+\tau V_{e} y_{1}^{2}\right)\right]}+\frac{e^{y_{2} t}\left(1+y_{2} \tau\right)^{2}\left(1-V_{e} y_{2}\right)}{y_{2}\left[\left(1+y_{2} \tau\right)^{2}\left(M+V_{e}\right)+l M\left(1+\tau V_{e} y_{2}^{2}\right)\right]}\right\} \\
& +\frac{G_{m}\left(\mathrm{R}_{2}-1\right) e^{-a t}}{\left(1+a V_{e}\right)\left(m_{2}^{2}-\alpha_{3}^{2}\right)}\left(\frac{\cosh \left(\alpha_{3} y\right)}{\cosh \left(\alpha_{3}\right)}-\frac{\cosh \left(m_{2} y\right)}{\cosh \left(m_{2}\right)}\right)+\pi M G_{m}\left(\mathrm{R}_{2}-1\right) \sum_{n=0}^{\infty} \frac{(2 n+1) \cos \left(\frac{2 n+1}{2} \pi y\right)}{(-1)^{n}\left[m_{2}^{2}+\left(\frac{2 n+1}{2} \pi\right)^{2}\right]} \\
& \times\left\{\frac{e^{y_{2} t}\left(1+y_{2} \tau\right)^{2}\left(1-V_{e} y_{2}\right)}{\left(y_{1}+a\right)\left[\left(1+y_{1} \tau\right)^{2}\left(M+V_{e}\right)+l M\left(1+\tau V_{e} y_{1}^{2}\right)\right]}+\frac{\left.V_{e} y_{1}\right)}{\left(y_{2}+a\right)\left[\left(1+y_{2} \tau\right)^{2}\left(M+V_{e}\right)+l M\left(1+\tau V_{e} y_{2}^{2}\right)\right]}\right\}
\end{aligned}
$$

and

$$
\begin{aligned}
& v=\frac{a_{0} \mathrm{e}^{-\mathrm{a}_{1} \mathrm{t}}}{\alpha_{1}{ }^{2}\left(1+\mathrm{a}_{1} V_{e}\right)\left(1-\mathrm{a}_{1} \tau\right)}\left(1-\frac{\cosh \left(\alpha_{1} y\right)}{\cosh \left(\alpha_{1}\right)}\right)+\frac{4 M a_{0}}{\pi} \sum_{n=0}^{\infty} \frac{\cos \left(\frac{2 n+1}{2} \pi y\right)}{(-1)^{n}(2 n+1)}\left\{\frac{1}{\left(y_{1}+a_{1}\right)}\right. \\
& \left.\times \frac{e^{y_{1} t}\left(1+y_{1} \tau\right)\left(1-V_{e} y_{1}\right)}{\left[\left(1+y_{1} \tau\right)^{2}\left(M+V_{e}\right)+l M\left(1+\tau V_{e} y_{1}{ }^{2}\right)\right]}+\frac{e^{y_{2} t}\left(1+y_{2} \tau\right)\left(1-V_{e} y_{2}\right)}{\left(y_{2}+a_{1}\right)\left[\left(1+y_{2} \tau\right)^{2}\left(M+V_{e}\right)+l M\left(1+\tau V_{e}{y_{2}}^{2}\right)\right]}\right\} \\
& +\pi M\left(G_{r}-\mathrm{R}_{1} G_{m}\right) \sum_{n=0}^{\infty} \frac{(2 n+1) \cos \left(\frac{2 n+1}{2} \pi y\right)}{(-1)^{n}\left[m_{1}{ }^{2}+\left(\frac{2 n+1}{2} \pi\right)^{2}\right]}\left\{\frac{e^{y_{1} t}\left(1+y_{1} \tau\right)\left(1-V_{e} y_{1}\right)}{y_{1}\left[\left(1+y_{1} \tau\right)^{2}\left(M+V_{e}\right)+l M\left(1+\tau V_{e} y_{1}^{2}\right)\right]}\right. \\
& \left.+\frac{e^{y_{2} t}\left(1+y_{2} \tau\right)\left(1-V_{e} y_{2}\right)}{y_{2}\left[\left(1+y_{2} \tau\right)^{2}\left(M+V_{e}\right)+l M\left(1+\tau V_{e} y_{2}^{2}\right)\right]}\right\}+\frac{\left(G_{r}-\mathrm{R}_{1} G_{m}\right)}{\left(m_{1}{ }^{2}-\alpha_{2}^{2}\right)}\left(\frac{\cosh \left(\alpha_{2} y\right)}{\cosh \left(\alpha_{2}\right)}-\frac{\cosh \left(m_{1} y\right)}{\cosh \left(m_{1}\right)}\right) \\
& -\pi M\left(G_{r}+\mathrm{R}_{2} G_{m}\right) \sum_{n=0}^{\infty} \frac{(2 n+1) \cos \left(\frac{2 n+1}{2} \pi y\right)}{(-1)^{n}\left[m_{1}{ }^{2}+\left(\frac{2 n+1}{2} \pi\right)^{2}\right]}\left\{\frac{e^{y_{1} t}\left(1+y_{1} \tau\right)\left(1-V_{e} y_{1}\right)}{\left(y_{1}+a\right)\left[\left(1+y_{1} \tau\right)^{2}\left(M+V_{e}\right)+l M\left(1+\tau V_{e} y_{1}{ }^{2}\right)\right]}\right. \\
& \left.+\frac{e^{y_{2} t}\left(1+y_{2} \tau\right)\left(1-V_{e} y_{2}\right)}{\left(y_{2}+a\right)\left[\left(1+y_{2} \tau\right)^{2}\left(M+V_{e}\right)+l M\left(1+\tau V_{e} y_{2}^{2}\right)\right]}\right\}-\frac{\left(G_{r}+\mathrm{R}_{2} G_{m}\right) e^{-a t}}{(1-\mathrm{a} \tau)\left(1+a V_{e}\right)\left(m_{1}{ }^{2}-\alpha_{3}{ }^{2}\right)} \\
& \times\left(\frac{\cosh \left(\alpha_{3} y\right)}{\cosh \left(\alpha_{3}\right)}-\frac{\cosh \left(m_{1} y\right)}{\cosh \left(m_{1}\right)}\right)+\frac{G_{m}\left(1+\mathrm{R}_{1}\right)}{\left(m_{2}{ }^{2}-\alpha_{2}{ }^{2}\right)}\left(\frac{\cosh \left(\alpha_{2} y\right)}{\cosh \left(\alpha_{2}\right)}-\frac{\cosh \left(m_{2} y\right)}{\cosh \left(m_{2}\right)}\right)+\pi M G_{m}\left(1+\mathrm{R}_{1}\right) \\
& \times \sum_{n=0}^{\infty} \frac{(2 n+1) \cos \left(\frac{2 n+1}{2} \pi y\right)}{(-1)^{n}\left[m_{2}^{2}+\left(\frac{2 n+1}{2} \pi\right)^{2}\right]}\left\{\frac{e^{y_{1} t}\left(1+y_{1} \tau\right)\left(1-V_{e} y_{1}\right)}{y_{1}\left[\left(1+y_{1} \tau\right)^{2}\left(M+V_{e}\right)+l M\left(1+\tau V_{e} y_{1}^{2}\right)\right]}\right. \\
& \left.+\frac{e^{y_{2} t}\left(1+y_{2} \tau\right)\left(1-V_{e} y_{2}\right)}{y_{2}\left[\left(1+y_{2} \tau\right)^{2}\left(M+V_{e}\right)+l M\left(1+\tau V_{e} y_{2}^{2}\right)\right]}\right\}+\frac{G_{m}\left(\mathrm{R}_{2}-1\right) e^{-a t}}{(1-\mathrm{a} \tau)\left(1+a V_{e}\right)\left(m_{2}{ }^{2}-\alpha_{3}^{2}\right)}\left(\frac{\cosh \left(\alpha_{3} y\right)}{\cosh \left(\alpha_{3}\right)}\right. \\
& \left.-\frac{\cosh \left(m_{2} y\right)}{\cosh \left(m_{2}\right)}\right)+\pi M G_{m}\left(\mathrm{R}_{2}-1\right) \sum_{n=0}^{\infty} \frac{(2 n+1) \cos \left(\frac{2 n+1}{2} \pi y\right)}{(-1)^{n}\left[m_{2}^{2}+\left(\frac{2 n+1}{2} \pi\right)^{2}\right]} \\
& \times\left\{\frac{e^{y_{1} t}\left(1+y_{1} \tau\right)\left(1-V_{e} y_{1}\right)}{\left(y_{1}+a\right)\left[\left(1+y_{1} \tau\right)^{2}\left(M+V_{e}\right)+l M\left(1+\tau V_{e} y_{1}^{2}\right)\right]}+\frac{e^{y_{2} t}\left(1+y_{2} \tau\right)\left(1-V_{e} y_{2}\right)}{\left(y_{2}+a\right)\left[\left(1+y_{2} \tau\right)^{2}\left(M+V_{e}\right)+l M\left(1+\tau V_{e} y_{2}{ }^{2}\right)\right]}\right\} .
\end{aligned}
$$

\section{Case - 2:}

Let $P_{0}(t)=a_{2}+a_{3} t$, where $a_{2}$ and $a_{3}$ are constants. The velocities of the fluid and dust particles are obtained by solving the equation (3.13) and (3.12) subjected to the boundary conditions (3.11) as follows

$\bar{u}=\frac{a_{2}}{s Q^{2}\left(1-V_{e} s\right)}\left(1-\frac{\cosh (Q y)}{\cosh (Q)}\right)+\frac{a_{3}}{s^{2} Q^{2}\left(1-V_{e} s\right)}\left(1-\frac{\cosh (Q y)}{\cosh (Q)}\right)+\frac{1}{\left(1-V_{e} s\right)\left(m_{1}{ }^{2}-Q^{2}\right)}$ 


$$
\begin{aligned}
& \times\left(\frac{\cosh (Q y)}{\cosh (Q)}-\frac{\cosh \left(m_{1} y\right)}{\cosh \left(m_{1}\right)}\right)\left(\frac{G_{r}-\mathrm{R}_{1} G_{m}}{\mathrm{~s}}-\frac{G_{r}+\mathrm{R}_{2} G_{m}}{\mathrm{~s}+\mathrm{a}}\right)+\frac{G_{m}}{\left(1-V_{e} s\right)\left(m_{2}{ }^{2}-Q^{2}\right)}\left(\frac{\cosh (Q y)}{\cosh (Q)}\right. \\
& \left.-\frac{\cosh \left(m_{2} y\right)}{\cosh \left(m_{2}\right)}\right)\left(\frac{1+\mathrm{R}_{1}}{\mathrm{~s}}-\frac{1-\mathrm{R}_{2}}{\mathrm{~s}+\mathrm{a}}\right)
\end{aligned}
$$

and

$$
\begin{aligned}
\bar{v}= & \frac{a_{2}}{s Q^{2}(1+s \tau)\left(1-V_{e} s\right)}\left(1-\frac{\cosh (Q y)}{\cosh (Q)}\right)+\frac{a_{3}}{s^{2} Q^{2}(1+s \tau)\left(1-V_{e} s\right)}\left(1-\frac{\cosh (Q y)}{\cosh (Q)}\right) \\
& +\frac{1}{(1+s \tau)\left(1-V_{e} s\right)\left(m_{1}{ }^{2}-Q^{2}\right)}\left(\frac{\cosh (Q y)}{\cosh (Q)}-\frac{\cosh \left(m_{1} y\right)}{\cosh \left(m_{1}\right)}\right)\left(\frac{G_{r}-\mathrm{R}_{1} G_{m}}{\mathrm{~s}}-\frac{G_{r}+\mathrm{R}_{2} G_{m}}{\mathrm{~s}+\mathrm{a}}\right) \\
& +\frac{G_{m}}{(1+s \tau)\left(1-V_{e} s\right)\left(m_{2}{ }^{2}-Q^{2}\right)}\left(\frac{\cosh (Q y)}{\cosh (Q)}-\frac{\cosh \left(m_{2} y\right)}{\cosh \left(m_{2}\right)}\right)\left(\frac{1+\mathrm{R}_{1}}{\mathrm{~s}}-\frac{1-\mathrm{R}_{2}}{\mathrm{~s}+\mathrm{a}}\right)
\end{aligned}
$$

Applying the inverse Laplace transform to the equations (3.16) and (3.17), $u$ and $v$ are obtained as

$$
\begin{aligned}
& u=\left[a_{2} M+a_{3} M t-a_{3} M^{2}(1+l)\right]\left(1-\frac{\cosh \left(\alpha_{2} y\right)}{\cosh \left(\alpha_{2}\right)}\right)+\frac{4 M a_{2}}{\pi} \sum_{n=0}^{\infty} \frac{\cos \left(\frac{2 n+1}{2} \pi y\right)}{(-1)^{n}(2 n+1)} \\
& \times\left\{\frac{e^{y_{1} t}\left(1+y_{1} \tau\right)^{2}\left(1-V_{e} y_{1}\right)}{y_{1}\left[\left(M+V_{e}\right)\left(1+y_{1} \tau\right)^{2}+l M\left(1+\tau V_{e} y_{1}^{2}\right)\right]}+\frac{e^{y_{2} t}\left(1+y_{2} \tau\right)^{2}\left(1-V_{e} y_{2}\right)}{y_{2}\left[\left(M+V_{e}\right)\left(1+y_{2} \tau\right)^{2}+l M\left(1+\tau V_{e} y_{2}^{2}\right)\right]}\right\} \\
& +\frac{a_{3} \sqrt{M}\left(M+V_{e}+M l\right)}{2 \cosh \left(\alpha_{2}\right)}\left(\frac{\sinh \left(\alpha_{2}\right) \cosh \left(\alpha_{2} y\right)}{\cosh \left(\alpha_{2}\right)}-y \sinh \left(\alpha_{2} y\right)\right)+\frac{4 M a_{3}}{\pi} \sum_{n=0}^{\infty} \frac{\cos \left(\frac{2 n+1}{2} \pi y\right)}{(-1)^{n}(2 n+1)} \\
& \times\left\{\frac{e^{y_{1} t}\left(1+y_{1} \tau\right)^{2}\left(1-V_{e} y_{1}\right)}{y_{1}{ }^{2}\left[\left(M+V_{e}\right)\left(1+y_{1} \tau\right)^{2}+l M\left(1+\tau V_{e} y_{1}^{2}\right)\right]}+\frac{e^{y_{2} t}\left(1+y_{2} \tau\right)^{2}\left(1-V_{e} y_{2}\right)}{y_{2}{ }^{2}\left[\left(M+V_{e}\right)\left(1+y_{2} \tau\right)^{2}+l M\left(1+\tau V_{e} y_{2}^{2}\right)\right]}\right\} \\
& +\pi M\left(G_{r}-\mathrm{R}_{1} G_{m}\right) \sum_{n=0}^{\infty} \frac{(2 n+1) \cos \left(\frac{2 n+1}{2} \pi y\right)}{(-1)^{n}\left[m_{1}{ }^{2}+\left(\frac{2 n+1}{2} \pi\right)^{2}\right]}\left\{\frac{e^{y_{1} t}\left(1+y_{1} \tau\right)^{2}\left(1-V_{e} y_{1}\right)}{y_{1}\left[\left(M+V_{e}\right)\left(1+y_{1} \tau\right)^{2}+l M\left(1+\tau V_{e} y_{1}{ }^{2}\right)\right]}\right. \\
& \left.+\frac{e^{y_{2} t}\left(1+y_{2} \tau\right)^{2}\left(1-V_{e} y_{2}\right)}{y_{2}\left[\left(M+V_{e}\right)\left(1+y_{2} \tau\right)^{2}+l M\left(1+\tau V_{e} y_{2}^{2}\right)\right]}\right\}+\frac{\left(G_{r}-\mathrm{R}_{1} G_{m}\right)}{\left(m_{1}{ }^{2}-\alpha_{2}{ }^{2}\right)}\left(\frac{\cosh \left(\alpha_{2} y\right)}{\cosh \left(\alpha_{2}\right)}-\frac{\cosh \left(m_{1} y\right)}{\cosh \left(m_{1}\right)}\right) \\
& -\pi M\left(G_{r}+\mathrm{R}_{2} G_{m}\right) \sum_{n=0}^{\infty} \frac{(2 n+1) \cos \left(\frac{2 n+1}{2} \pi y\right)}{(-1)^{n}\left[m_{1}{ }^{2}+\left(\frac{2 n+1}{2} \pi\right)^{2}\right]}\left\{\frac{e^{y_{1} t}\left(1+y_{1} \tau\right)^{2}\left(1-V_{e} y_{1}\right)}{\left(y_{1}+a\right)\left[\left(M+V_{e}\right)\left(1+y_{1} \tau\right)^{2}+l M\left(1+\tau V_{e} y_{1}{ }^{2}\right)\right]}\right. \\
& \left.+\frac{e^{y_{2} t}\left(1+y_{2} \tau\right)^{2}\left(1-V_{e} y_{2}\right)}{\left(y_{2}+a\right)\left[\left(M+V_{e}\right)\left(1+y_{2} \tau\right)^{2}+l M\left(1+\tau V_{e} y_{2}^{2}\right)\right]}\right\}-\frac{\left(G_{r}+\mathrm{R}_{2} G_{m}\right) e^{-a t}}{\left(1+a V_{e}\right)\left(m_{1}{ }^{2}-\alpha_{3}{ }^{2}\right)}\left(\frac{\cosh \left(\alpha_{3} y\right)}{\cosh \left(\alpha_{3}\right)}-\frac{\cosh \left(m_{1} y\right)}{\cosh \left(m_{1}\right)}\right) \\
& +\frac{G_{m}\left(1+\mathrm{R}_{1}\right)}{\left(m_{2}{ }^{2}-\alpha_{2}{ }^{2}\right)}\left(\frac{\cosh \left(\alpha_{2} y\right)}{\cosh \left(\alpha_{2}\right)}-\frac{\cosh \left(m_{2} y\right)}{\cosh \left(m_{2}\right)}\right)+\pi M G_{m}\left(1+\mathrm{R}_{1}\right) \sum_{n=0}^{\infty} \frac{(2 n+1) \cos \left(\frac{2 n+1}{2} \pi y\right)}{(-1)^{n}\left[m_{2}^{2}+\left(\frac{2 n+1}{2} \pi\right)^{2}\right]} \\
& \times\left\{\frac{e^{y_{1} t}\left(1+y_{1} \tau\right)^{2}\left(1-V_{e} y_{1}\right)}{y_{1}\left[\left(M+V_{e}\right)\left(1+y_{1} \tau\right)^{2}+l M\left(1+\tau V_{e} y_{1}^{2}\right)\right]}+\frac{e^{y_{2} t}\left(1+y_{2} \tau\right)^{2}\left(1-V_{e} y_{2}\right)}{y_{2}\left[\left(M+V_{e}\right)\left(1+y_{2} \tau\right)^{2}+l M\left(1+\tau V_{e} y_{2}^{2}\right)\right]}\right\} \\
& +\frac{G_{m}\left(\mathrm{R}_{2}-1\right) e^{-a t}}{\left(1+a V_{e}\right)\left(m_{2}^{2}-\alpha_{3}^{2}\right)}\left(\frac{\cosh \left(\alpha_{3} y\right)}{\cosh \left(\alpha_{3}\right)}-\frac{\cosh \left(m_{2} y\right)}{\cosh \left(m_{2}\right)}\right)+\pi M G_{m}\left(\mathrm{R}_{2}-1\right) \\
& \times \sum_{n=0}^{\infty} \frac{(2 n+1) \cos \left(\frac{2 n+1}{2} \pi y\right)}{(-1)^{n}\left[m_{2}^{2}+\left(\frac{2 n+1}{2} \pi\right)^{2}\right]}\left\{\frac{e^{y_{1} t}\left(1+y_{1} \tau\right)^{2}\left(1-V_{e} y_{1}\right)}{\left(y_{1}+a\right)\left[\left(M+V_{e}\right)\left(1+y_{1} \tau\right)^{2}+l M\left(1+\tau V_{e} y_{1}^{2}\right)\right]}\right. \\
& \left.+\frac{e^{y_{2} t}\left(1+y_{2} \tau\right)^{2}\left(1-V_{e} y_{2}\right)}{\left(y_{2}+a\right)\left[\left(M+V_{e}\right)\left(1+y_{2} \tau\right)^{2}+l M\left(1+\tau V_{e} y_{2}^{2}\right)\right]}\right\}
\end{aligned}
$$


and

$$
\begin{aligned}
& v=\left[a_{2} M+a_{3} M(t-\tau)-a_{3} M^{2}(1+l)\right]\left(1-\frac{\cosh \left(\alpha_{2} y\right)}{\cosh \left(\alpha_{2}\right)}\right)+\frac{4 M a_{2}}{\pi} \sum_{n=0}^{\infty} \frac{\cos \left(\frac{2 n+1}{2} \pi y\right)}{(-1)^{n}(2 n+1)} \\
& \times\left\{\frac{e^{y_{1} t}\left(1+y_{1} \tau\right)\left(1-V_{e} y_{1}\right)}{y_{1}\left[\left(M+V_{e}\right)\left(1+y_{1} \tau\right)^{2}+l M\left(1+\tau V_{e} y_{1}^{2}\right)\right]}+\frac{e^{y_{2} t}\left(1+y_{2} \tau\right)\left(1-V_{e} y_{2}\right)}{y_{2}\left[\left(M+V_{e}\right)\left(1+y_{2} \tau\right)^{2}+l M\left(1+\tau V_{e}{y_{2}}^{2}\right)\right]}\right\} \\
& +\frac{a_{3} \sqrt{M}\left(M+V_{e}+M l\right)}{2 \cosh \left(\alpha_{2}\right)}\left(\frac{\sinh \left(\alpha_{2}\right) \cosh \left(\alpha_{2} y\right)}{\cosh \left(\alpha_{2}\right)}-y \sinh \left(\alpha_{2} y\right)\right)+\frac{4 M a_{3}}{\pi} \sum_{n=0}^{\infty} \frac{\cos \left(\frac{2 n+1}{2} \pi y\right)}{(-1)^{n}(2 n+1)} \\
& \times\left\{\frac{e^{y_{1} t}\left(1+y_{1} \tau\right)\left(1-V_{e} y_{1}\right)}{y_{1}{ }^{2}\left[\left(M+V_{e}\right)\left(1+y_{1} \tau\right)^{2}+l M\left(1+\tau V_{e} y_{1}^{2}\right)\right]}+\frac{e^{y_{2} t}\left(1+y_{2} \tau\right)\left(1-V_{e} y_{2}\right)}{y_{2}^{2}\left[\left(M+V_{e}\right)\left(1+y_{2} \tau\right)^{2}+l M\left(1+\tau V_{e} y_{2}^{2}\right)\right]}\right\} \\
& +\pi M\left(G_{r}-\mathrm{R}_{1} G_{m}\right) \sum_{n=0}^{\infty} \frac{(2 n+1) \cos \left(\frac{2 n+1}{2} \pi y\right)}{(-1)^{n}\left[m_{1}{ }^{2}+\left(\frac{2 n+1}{2} \pi\right)^{2}\right]}\left\{\frac{e^{y_{1} t}\left(1+y_{1} \tau\right)\left(1-V_{e} y_{1}\right)}{y_{1}\left[\left(M+V_{e}\right)\left(1+y_{1} \tau\right)^{2}+l M\left(1+\tau V_{e} y_{1}{ }^{2}\right)\right]}\right. \\
& \left.+\frac{e^{y_{2} t}\left(1+y_{2} \tau\right)\left(1-V_{e} y_{2}\right)}{y_{2}\left[\left(M+V_{e}\right)\left(1+y_{2} \tau\right)^{2}+l M\left(1+\tau V_{e} y_{2}^{2}\right)\right]}\right\}+\frac{\left(G_{r}-\mathrm{R}_{1} G_{m}\right)}{\left(m_{1}{ }^{2}-\alpha_{2}^{2}\right)}\left(\frac{\cosh \left(\alpha_{2} y\right)}{\cosh \left(\alpha_{2}\right)}-\frac{\cosh \left(m_{1} y\right)}{\cosh \left(m_{1}\right)}\right) \\
& -\pi M\left(G_{r}+\mathrm{R}_{2} G_{m}\right) \sum_{n=0}^{\infty} \frac{(2 n+1) \cos \left(\frac{2 n+1}{2} \pi y\right)}{(-1)^{n}\left[m_{1}^{2}+\left(\frac{2 n+1}{2} \pi\right)^{2}\right]}\left\{\frac{e^{y_{1} t}\left(1+y_{1} \tau\right)\left(1-V_{e} y_{1}\right)}{\left(y_{1}+a\right)\left[\left(M+V_{e}\right)\left(1+y_{1} \tau\right)^{2}+l M\left(1+\tau V_{e} y_{1}^{2}\right)\right]}\right. \\
& \left.+\frac{e^{y_{2} t}\left(1+y_{2} \tau\right)\left(1-V_{e} y_{2}\right)}{\left(y_{2}+a\right)\left[\left(M+V_{e}\right)\left(1+y_{2} \tau\right)^{2}+l M\left(1+\tau V_{e} y_{2}{ }^{2}\right)\right]}\right\}-\frac{\left(G_{r}+\mathrm{R}_{2} G_{m}\right) e^{-a t}}{(1-\mathrm{a} \tau)\left(1+a V_{e}\right)\left(m_{1}{ }^{2}-\alpha_{3}{ }^{2}\right)}\left(\frac{\cosh \left(\alpha_{3} y\right)}{\cosh \left(\alpha_{3}\right)}\right. \\
& \left.-\frac{\cosh \left(m_{1} y\right)}{\cosh \left(m_{1}\right)}\right)+\frac{G_{m}\left(1+\mathrm{R}_{1}\right)}{\left(m_{2}{ }^{2}-\alpha_{2}{ }^{2}\right)}\left(\frac{\cosh \left(\alpha_{2} y\right)}{\cosh \left(\alpha_{2}\right)}-\frac{\cosh \left(m_{2} y\right)}{\cosh \left(m_{2}\right)}\right)+\pi M G_{m}\left(1+\mathrm{R}_{1}\right) \sum_{n=0}^{\infty} \frac{(2 n+1) \cos \left(\frac{2 n+1}{2} \pi y\right)}{(-1)^{n}\left[m_{2}^{2}+\left(\frac{2 n+1}{2} \pi\right)^{2}\right]} \\
& \times\left\{\frac{e^{y_{1} t}\left(1+y_{1} \tau\right)\left(1-V_{e} y_{1}\right)}{y_{1}\left[\left(M+V_{e}\right)\left(1+y_{1} \tau\right)^{2}+l M\left(1+\tau V_{e} y_{1}^{2}\right)\right]}+\frac{e^{y_{2} t}\left(1+y_{2} \tau\right)\left(1-V_{e} y_{2}\right)}{y_{2}\left[\left(M+V_{e}\right)\left(1+y_{2} \tau\right)^{2}+l M\left(1+\tau V_{e}{y_{2}}^{2}\right)\right]}\right\} \\
& +\frac{G_{m}\left(\mathrm{R}_{2}-1\right) e^{-a t}}{(1-\mathrm{a} \tau)\left(1+a V_{e}\right)\left(m_{2}{ }^{2}-\alpha_{3}{ }^{2}\right)}\left(\frac{\cosh \left(\alpha_{3} y\right)}{\cosh \left(\alpha_{3}\right)}-\frac{\cosh \left(m_{2} y\right)}{\cosh \left(m_{2}\right)}\right)+\pi M G_{m}\left(\mathrm{R}_{2}-1\right) \sum_{n=0}^{\infty} \frac{(2 n+1) \cos \left(\frac{2 n+1}{2} \pi y\right)}{(-1)^{n}\left[m_{2}{ }^{2}+\left(\frac{2 n+1}{2} \pi\right)^{2}\right]} \\
& \times\left\{\frac{e^{y_{1} t}\left(1+y_{1} \tau\right)\left(1-V_{e} y_{1}\right)}{\left(y_{1}+a\right)\left[\left(M+V_{e}\right)\left(1+y_{1} \tau\right)^{2}+l M\left(1+\tau V_{e} y_{1}{ }^{2}\right)\right]}+\frac{e^{y_{2} t}\left(1+y_{2} \tau\right)\left(1-V_{e} y_{2}\right)}{\left(y_{2}+a\right)\left[\left(M+V_{e}\right)\left(1+y_{2} \tau\right)^{2}+l M\left(1+\tau V_{e} y_{2}^{2}\right)\right]}\right\}
\end{aligned}
$$

where

$R_{1}=\frac{T_{d} m_{1}^{2}}{m_{1}{ }^{2}-c_{r} S_{c}}, \quad R_{2}=-\frac{T_{d} m_{1}{ }^{2}}{m_{1}{ }^{2}+a S_{c}-c_{r} S_{c}}, \quad a_{11}=\tau M-\left(\frac{2 n+1}{2}\right)^{2} \pi^{2} \tau M V_{e}$,

$b_{11}=M+l M+\tau+\left(\frac{2 n+1}{2}\right)^{2} \pi^{2} M\left(\tau-V_{e}\right), \quad c_{11}=1+\left(\frac{2 n+1}{2}\right)^{2} \pi^{2} M$,

$y_{1}=\frac{-b_{11}+\sqrt{b_{11}{ }^{2}-4 a_{11} c_{11}}}{2 a_{11}}, \quad y_{2}=\frac{-b_{11}-\sqrt{{b_{11}}^{2}-4 a_{11} c_{11}}}{2 a_{11}}$,

$\alpha_{1}^{2}=\frac{1}{1+a_{1} V_{e}}\left(-a_{1}-\frac{a_{1} l}{1-a_{1} \tau}+\frac{1}{M}\right), \quad \alpha_{2}^{2}=\frac{1}{M}$ and $\alpha_{3}^{2}=\frac{1}{1+a V_{e}}\left(-a-\frac{a l}{1-a \tau}+\frac{1}{M}\right)$.

\section{Conclusions:}

The analytical solutions are obtained for temperature, concentration and velocity profiles. The following interpretations are evident from the graphs, 
- Fig. (2) and (3) shows the effect of thermal diffusion parameter $\left(\mathrm{T}_{\mathrm{d}}\right)$ and the heat source or sink parameter $\mathrm{H}_{\mathrm{s}}$ on temperature of the fluid. It is observed that $\mathrm{T}_{d}$ and $\mathrm{H}_{\mathrm{s}}$ decreases with increase of temperature.

- Fig. (4) and (5) confirms that concentration of the fluid increases when $\mathrm{T}_{\mathrm{d}}$ decreases and $\mathrm{Schmit}$ number $\left(\mathrm{S}_{\mathrm{c}}\right)$ increases respectively.

- Fig. (6) to (9) represents velocity profile for fluid and dust particles respectively.

- Fig. (6) and (7) depicts that the velocity increases as $\mathrm{T}_{\mathrm{d}}$ decreases. The soret effect is observed on velocities of fluid and dust particles when chemical reaction parameter $\left(\mathrm{c}_{\mathrm{r}}\right)$ is considered.

- Fig. (8) and (9) portrays the same progression as in Fig. (6) and (7).

- If the dust particle is very fine i.e., mass of the dust particles is negligibly small then the relaxation time of dust particles decreases and ultimately as $\tau \rightarrow 0$ the velocities of both fluid and dust particles will be the same.

- The fluid particles will reach the steady state earlier than the dust particles. This is due to the fact that time dependent pressure gradient is directly exerted on the fluid.
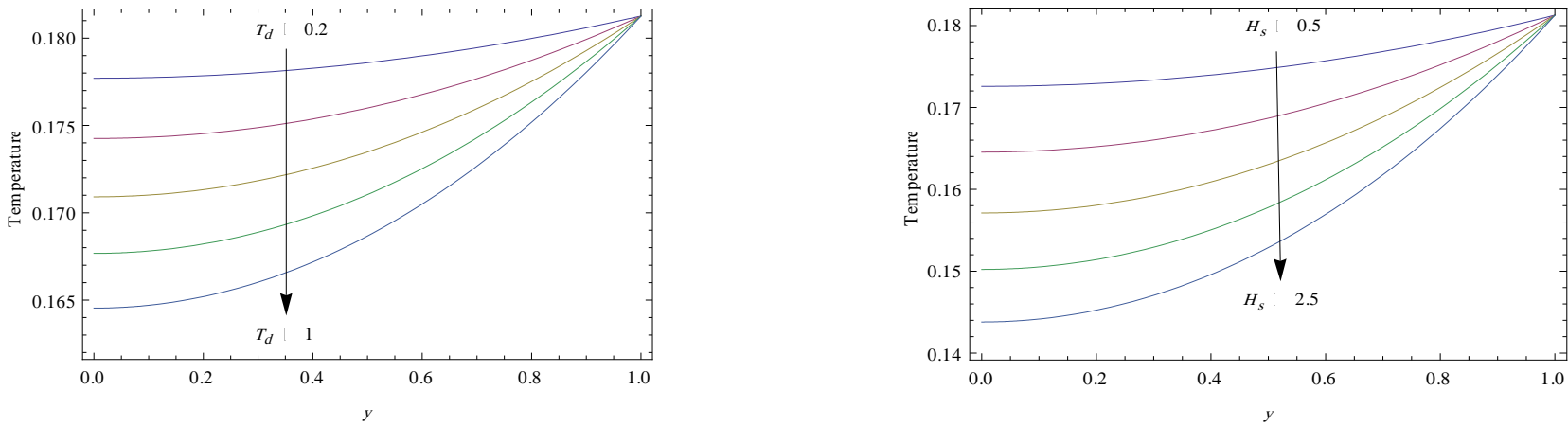

Fig. 2,3: Variation of the temperature with $T_{d}$ and $H_{s}$.
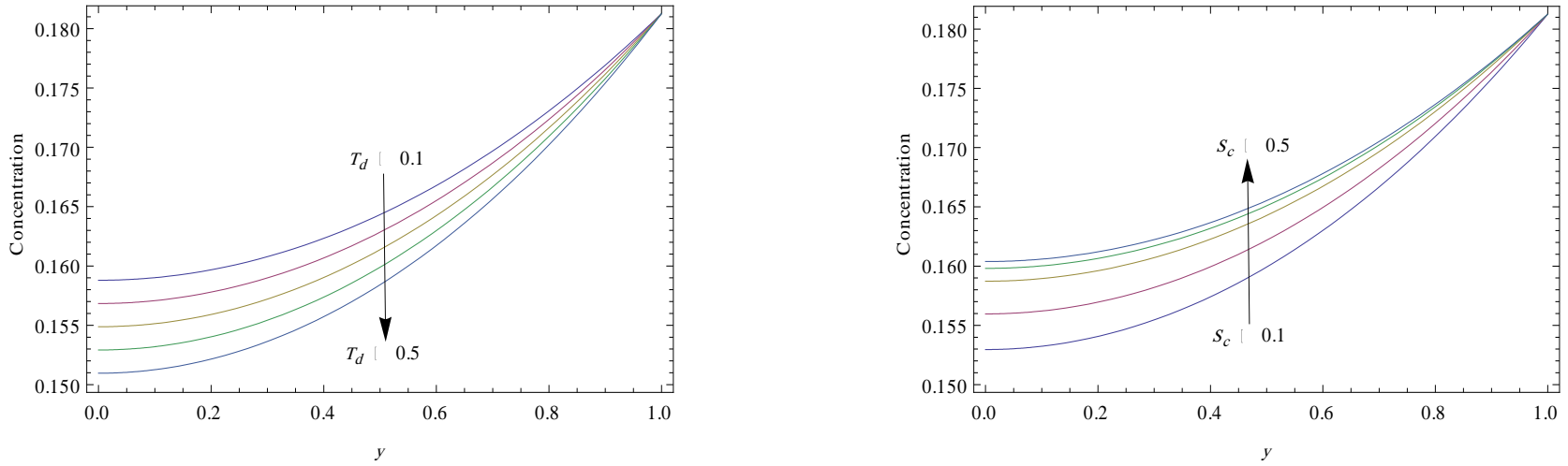

Fig. 4,5: Variation of the concentration with $T_{d}$ and $S_{c}$.
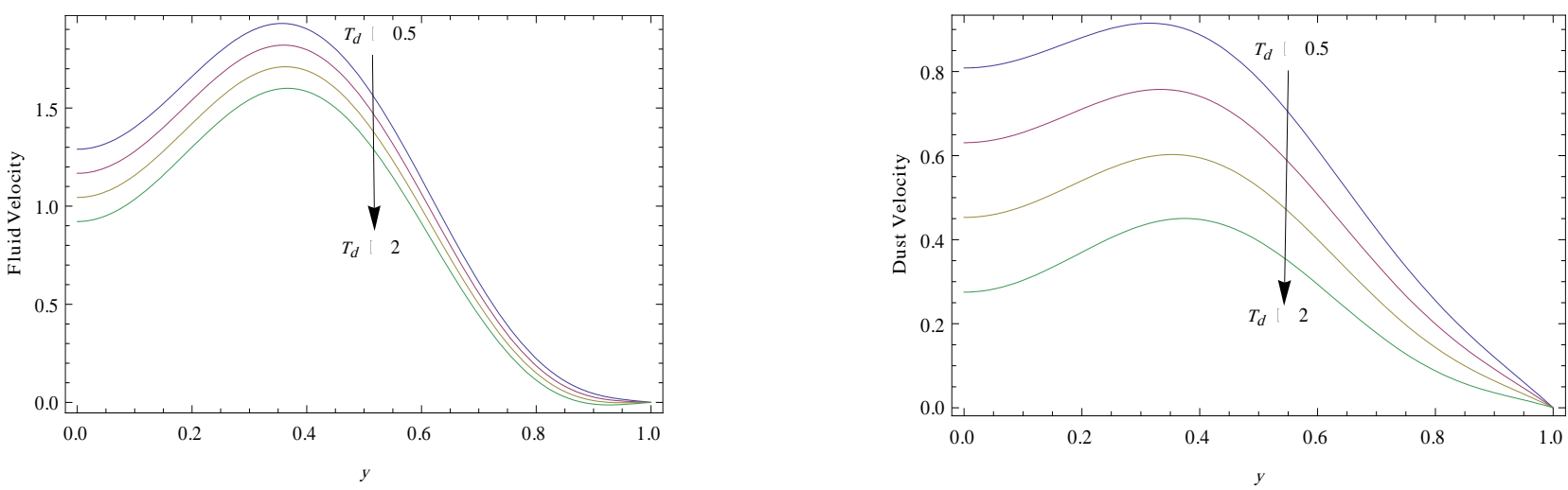

Fig. 6: Variation of the fluid and dust phase velocity with $T_{d}$ (Case-1). 

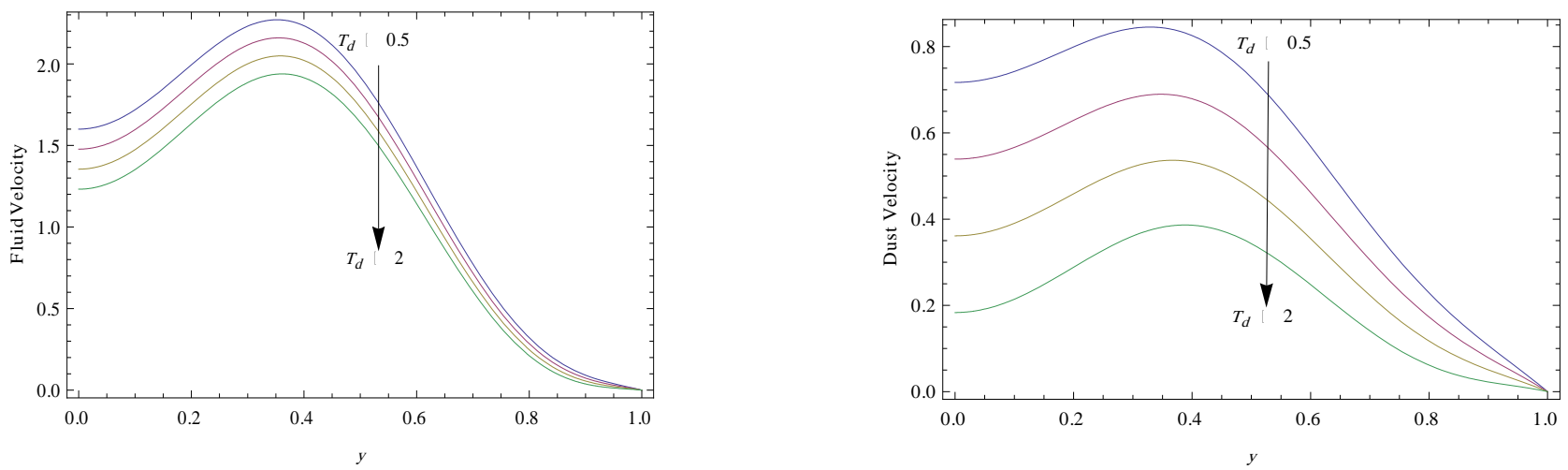

Fig. 7: Variation of the fluid and dust phase velocity with $T_{d}$ (Case-2).
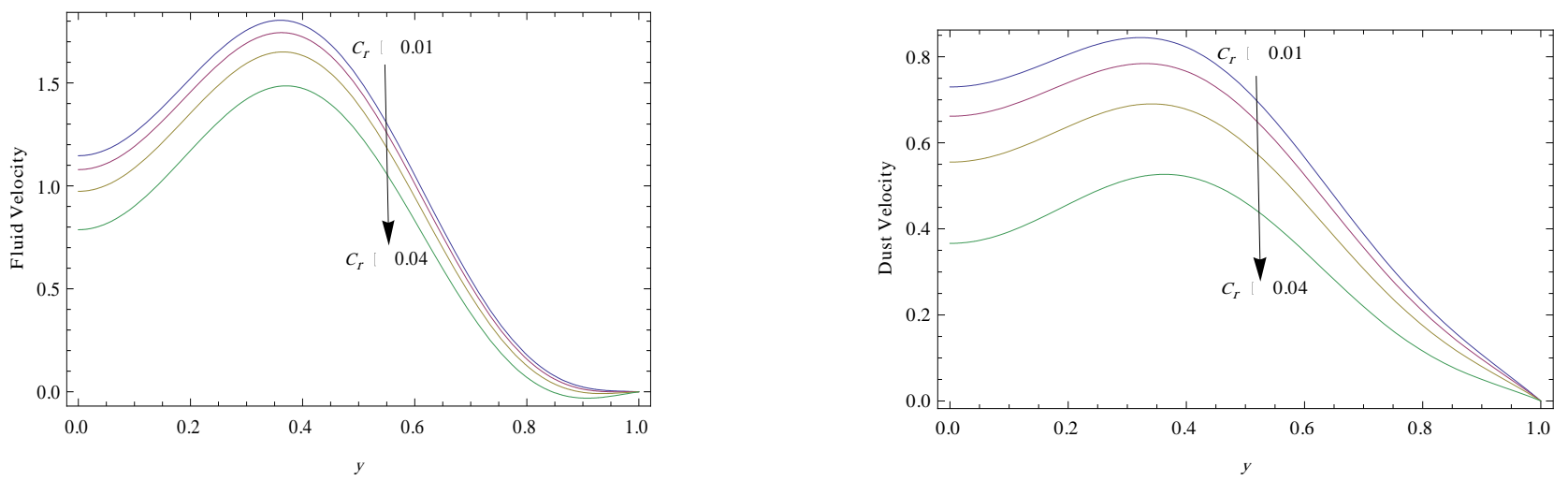

Fig. 8: Variation of the fluid and dust phase velocity with $c_{r}$ (Case-1).
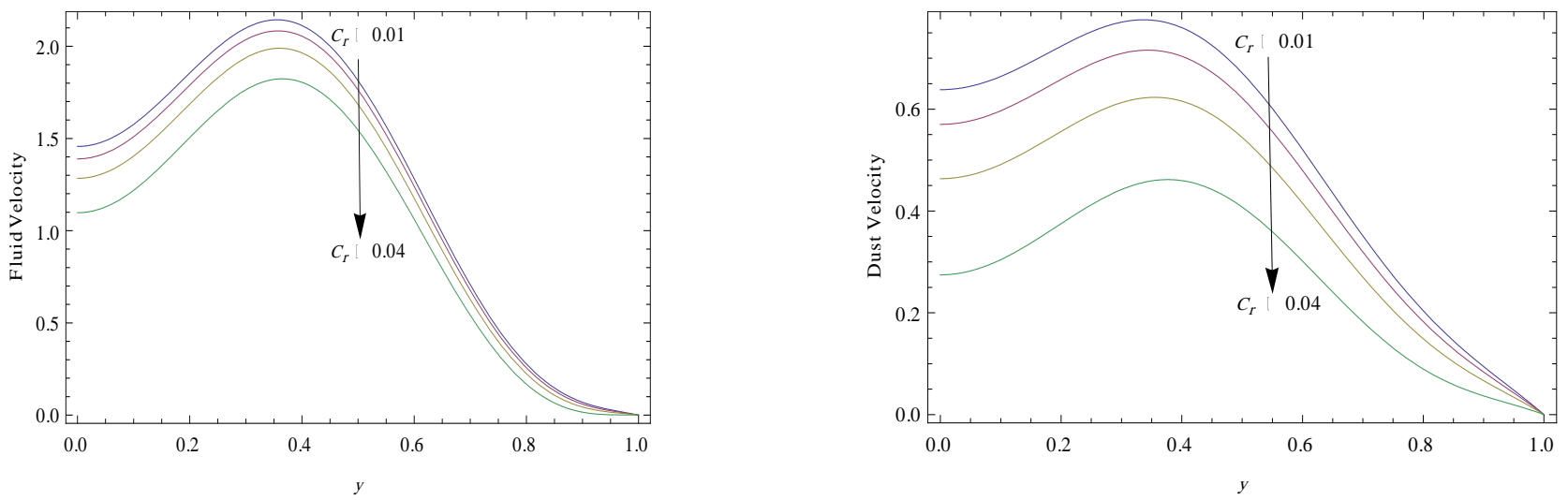

Fig. 9: Variation of the fluid and dust phase velocity with $c_{r}$ (Case-2).

The values considered for above graphs are $c_{r}=0.4, a=0.2, H_{s}=0.2, t=1, P_{r}=0.4, S_{c}=0.6, M=10, l=0.1, n=2$, $D=1, c_{1}=0.5, V_{e}=0.3, \tau=0.9, G_{m}=5, G_{r}=10, a_{0}=2.5, a_{1}=0.3, a_{2}=2.5, a_{3}=0.2$ and $m_{2}=1.5$. 


\section{References}

[1] P.L. Chamber and J.D. Young, On diffusion of a chemically reactive species in a laminar boundary layer flow, Physics of Fluids, Vol.1, 1958, pp.48-54.

[2] P.G. Saffman, On the stability of laminar flow of a dusty gas, Journal of Fluid Mechanics, Vol.13(1), 1962, pp.120-129.

[3] D.H. Michael and P.W. Norey, The laminar flow of a dusty gas between rotating cylinders, The Quarterly Journal of Mechanics and Applied Mathematics, Vol.21, 1968, pp.375-388.

[4] A. Rapus and C.P. Perdikis, Oscillating flow through a porous medium by the presence of free convective flow, International Journal of Engineering Science, Vol.23, 1985, pp.51-55.

[5] N.P. Singh, A.K. Singh and M.K. Yadav, Acta ciencia indica, Vol. XXVIII M(1), 2002, pp.089.

[6] D. Kumar and R.K. Srivastava, Effects of chemical reaction on MHD flow of dusty visco-elastic (Walter's liquid model-B) liquid with heat source/sink, Proceedings of National Seminar on Mathematics and Computer Science, Meerut, 2005, pp.105-112.

[7] I.U. Mbeledogu and A. Ogulu, Heat and mass transfer of an unsteady MHD natural convection flow of a rotating fluid past a vertical porous flat plate in the presence of radiative heat transfer, International Journal of Heat and Mass Transfer, Vol.50(9-10), 2007, pp.1902-1908.

[8] P.M. Patil and P.S. Kulkarni, Effects of chemical reaction on free convective flow of a polar fluid through a porous medium in the presence of internal heat generation, International Journal of Thermal Sciences, Vol.47(8), 2008, pp.1043-1054.

[9] E. Osalusi, J. Side and R. Harris, Thermal diffusion and thermo effect on combined heat and mass transfer of a steady MHD convective and slip flow due to a rotating disk with viscous dissipation and ohmic heating, International Communications in Heat and Mass Transfer, Vol.35(8), 2008, pp.908-915.

[10] A.A. Afify, Similarity solution in MHD: Effects of thermal diffusion and diffusion thermo on free convective heat and mass transfer over a stretching surface considering suction or injection, Communications in Nonlinear Science and Numerical Simulation, Vol.14(5), 2009, pp.2202-2214.

[11] O.A. Beg, A.Y. Bakier and V.R. Prasad, Numerical study of free convection magneto hydrodynamics heat and mass transfer from a stretching surface to a saturated porous medium with soret and dufour effects, Computational Materials Science, Vol.46(1), 2009, pp.57-65.

[12] K.R. Madhura, B.J. Gireesha and C.S. Bagewadi, Flow of an unsteady dusty fluid through porous media in a channel of triangular crosssection, International Review of Physics, Vol.4(6), Dec. 2010, pp. 315.

[13] M.M. Nandeppanavar, M.S. Abel and Tawade, Heat transfer in a Walter's liquid B fluid over an impermeable stretching sheet with nonuniform heat source/sink and elastic deformation, Communications in Nonlinear Science and Numerical Simulation, Vol.15(7), 2010, pp.1791-1802.

[14] Om Prakash, Devendra Kumar, Y.K. Dwivedi, Effects of thermal diffusion and chemical reaction on MHD flow of dusty visco-elastic (Walter's liquid model-B) fluid, J.Electromagnetic Analysis and Applications, Vol.2, 2010, pp.581-587.

[15] R. Sharma, R. Bhargava and P. Bhargava, A numerical solution of steady MHD convective heat and mass transfer on a semi infinite vertical porous moving plate using element free galerkin method, Computational Materials Science, Vol.48(3), 2010, pp.537-543.

[16] B.J. Gireesha, K.R. Madhura and C.S. Bagewadi, Flow of an unsteady dusty fluid through porous media between horizontal plate and a long wavy wall, International Review of Physics, Vol.6(1), Feb. 2012, pp.36. 